\title{
Advances in Yield Calibration of Scintillators
}

\author{
Johan T. M. de Haas and Pieter Dorenbos, Member, IEEE
}

\begin{abstract}
By means of a photomultiplier tube, a Si-photodiode, and a Si-avalanche photodiode, the absolute scintillation yield of recently developed $\mathrm{LaBr}_{3}: \mathrm{Ce}, \mathrm{LaCl}_{3}: \mathrm{Ce}$, and $(\mathrm{Lu}, \mathrm{Y})_{2} \mathrm{SiO}_{5}: \mathrm{Ce}$ scintillators and traditional $\mathrm{Lu}_{2} \mathrm{SiO}_{5}: \mathrm{Ce}, \mathrm{Bi}_{4} \mathrm{Ge}_{3} \mathrm{O}_{12}$, NaI:Tl CsI:TI, and CsI:Na scintillators were determined. These are all well known scintillators that cover emission wavelengths from $250 \mathrm{~nm}$ to $750 \mathrm{~nm}$. By comparing the scintillation yield independently measured with the three different photon detectors reliable yield values are obtained.
\end{abstract}

Index Terms-Absolute scintillation yield, gamma detectors, photodiode readout, scintillators.

\section{INTRODUCTION}

$\mathbf{S}^{\mathrm{C}}$ CINTILLATORS are widely used to detect the energy of gamma radiation. An important parameter that determines energy resolution but also timing resolution is the absolute number of photons emitted per $\mathrm{MeV}$ of gamma ray energy absorbed. The photon collection efficiency, the effective quantum efficiency and the gain of the photon detector all need to be known precisely in order to derive the photon yield from the output signals of the photon detector. Various important papers have appeared in the past. For example the early work by Sakai in 1987 [1] and Holl et al. in 1988 [2], and later by Moszyński et al. in 1997 [3]. Calibrations are based on the quantum efficiency of the photon detector, and a quantum efficiency curve provided by the manufacturer is usually used. However, the circumstances under which scintillators are used are very different from the circumstances under which photon detector quantum efficiencies are determined. Scintillation photons reflected from the photon detector are back-reflected by the reflector around the scintillator, and this effectively enhances the quantum efficiency. This was demonstrated and taken into account by Holl et al. [2] in their studies to determine the absolute scintillator yield with photodiodes (PDs). They also suggested that such correction should be made when calibrations are performed with photomultiplier tubes (PMTs). Moszyński et al. in 1997 [3] adopted the method of Holl et $a l$. in their studies with PDs. Based on the properties of quartz alone they assumed the PMT to have $8 \%$ reflectivity. The reflectivity of the photocathode material was not taken into account. In [4] we actually measured the reflectivity of the PMT quartz window and photocathode and found reflection values as large as $35 \%$. Correcting for reflectivity, we re-determined the

Manuscript received May 31, 2007; revised November 1, 2007.

The authors are with the Delft University of Technology, Faculty of Applied Sciences, Mekelweg 15, 2629JB, Delft, The Netherlands (e-mail: j.t.m.dehaas@tudelft.nl; p.dorenbos@tudelft.nl).

Color versions of one or more of the figures in this paper are available online at http://ieeexplore.ieee.org.

Digital Object Identifier 10.1109/TNS.2008.922819 scintillation yield of CsI:Tl, CaI:Na, $\mathrm{Bi}_{4} \mathrm{Ge}_{3} \mathrm{O}_{12}$ (BGO), and $\mathrm{YAlO}_{3}$ :Ce with two PMTs and two APDs. The PMT results turned out to be about $10-15 \%$ smaller than those with the APDs. In a following paper [5] we addressed the aspect of gain determination of a PMT. The gain should be determined from the mean value of the so-called single photoelectron response (SER) instead of, what we [4] and others [1] and [3] commonly did, the maximum of the SER. The scintillation yield of $\mathrm{LaCl}_{3}: 10 \% \mathrm{Ce}$ and $\mathrm{LaBr}_{3}: 5 \% \mathrm{Ce}$ were determined, and revised values for the light yield of the samples from [4] were presented. Good agreement between PMT and APD results was obtained. Gierlik et al. [6] do not agree with our methods; especially the magnitude of the reflectivity correction used by us is much too large in their opinion. However, their arguments, are based on experiments that do not properly represent our ideas on the physics behind the reflectivity correction.

In this work we again determine the absolute scintillation yield of CsI:Tl, CsI:Na, $\mathrm{Bi}_{4} \mathrm{Ge}_{3} \mathrm{O}_{12}$ (BGO), $\mathrm{LaCl}_{3}: 10 \% \mathrm{Ce}$ and $\mathrm{LaBr}_{3}: 5 \% \mathrm{Ce}$ but on a new set of small samples. In addition samples of $\mathrm{NaI}: \mathrm{Tl}, \mathrm{Lu}_{2} \mathrm{SiO}_{5}: \mathrm{Ce}(\mathrm{LSO})$, and $(\mathrm{Lu}, \mathrm{Y})_{2} \mathrm{SiO}_{5}: \mathrm{Ce}$ (LYSO) were calibrated. We used three different photon detectors; the same PMTs and APD that were used in [4] and [5] and in addition a PD. The emission of the scintillators covers the wavelength range from $250 \mathrm{~nm}$ to $750 \mathrm{~nm}$. Special attention is given to photon losses in the Si dead layer of APDs and PDs. The results from the three different photon detectors provide a consistent set of scintillation light yield values.

\section{EXPERIMENTAL METHODS}

Table I shows the scintillation crystals that were studied in this work. The crystals were kindly provided by Saint Gobain Crystals \& Detectors in France, by Scionix in The Netherlands, and by Crystal Photonics in Florida, USA. All crystals are bare, i.e., they are not packed in sealed housings with window material. To minimize photon losses by self-absorption in the crystal we have chosen to study small samples of few $\mathrm{mm}$ thicknesses. The measured yields can then be regarded as the intrinsic absolute light output of the scintillation material.

The scintillator mounted on a photo-detector was always measured in complete darkness. Experiments on the hygroscopic CsI:Na, NaI:Tl, $\mathrm{LaCl}_{3}: 10 \% \mathrm{Ce}$, and $\mathrm{LaBr}_{3}: 5 \% \mathrm{Ce}$ were performed inside a drybox with less than $1 \mathrm{ppm}_{2} \mathrm{O}$. Scintillation pulse height spectra were recorded using a $662 \mathrm{keV}{ }^{137} \mathrm{Cs}$ gamma source. The photon detector output was pre-amplified and shaped with a shaping time of $10 \mu$ s by an ORTEC 672 spectroscopic amplifier.

\section{A. Photomultiplier Tube Readout}

We used two Hamamatsu type R1791 PMT (quartz window version of the R878 PMT) with a box-and-grid type of dynode 
TABLE I

COMPILATION OF SCINTILLATOR TYPES, SCINTILLATOR DiMENSIONS, SCINTILlator MANUfaCtURER TOGETHER WITH THE MEAN EMISSION WAVELENGTH $\left(\lambda_{\mathrm{M}}\right)$ AND THE WAVELENGTH RANGE OF EMISSION

\begin{tabular}{|c|c|c|c|c|}
\hline $\begin{array}{c}\text { SCINTILLATOR } \\
\text { TYPE }\end{array}$ & $\begin{array}{r}\lambda_{\mathrm{M}} \\
{[\mathrm{nm}]}\end{array}$ & $\begin{array}{c}\text { RANGE } \\
{[\mathrm{nm}]}\end{array}$ & $\begin{array}{c}\text { DIMENSIONS } \\
{\left[\mathrm{mm}^{3}\right]}\end{array}$ & PROVIDED BY: \\
\hline $\mathrm{LaCl}_{3}: 5 \% \mathrm{Ce}^{3+}$ & 350 & $315-400$ & $2 \times 2 \times 4$ & Saint Gobain \\
\hline $\mathrm{LaBr}_{3}: 10 \% \mathrm{Ce}^{3+}$ & 375 & $340-435$ & $3 \times 3 \times 4$ & Saint Gobain \\
\hline $\mathrm{NaI}: \mathrm{Tl}^{+}$ & 415 & $315-550$ & $\varnothing 8 \times 3$ & Saint Gobain \\
\hline $\mathrm{Lu}_{2} \mathrm{SiO}_{5}: \mathrm{Ce}^{3+}$ & 425 & $375-650$ & $3 \times 5 \times 6$ & $\begin{array}{l}\text { Crystal } \\
\text { Photonics }\end{array}$ \\
\hline$(\mathrm{Lu}, \mathrm{Y})_{2} \mathrm{SiO}_{5}: \mathrm{Ce}^{3+}$ & 425 & $370-630$ & $3 \times 3 \times 6$ & $\begin{array}{l}\text { Crystal } \\
\text { Photonics }\end{array}$ \\
\hline CsI: $\mathrm{Na}^{+}$ & 425 & $335-800$ & $\varnothing 6 \times 1$ & Scionix \\
\hline $\mathrm{Bi}_{4} \mathrm{Ge}_{3} \mathrm{O}_{12}$ & 510 & $340-800$ & $\varnothing 5 \times 1$ & Scionix \\
\hline $\mathrm{CsI}_{\mathrm{Tl}}^{+}$ & 560 & $250-700$ & $\varnothing 6 \times 1$ & Scionix \\
\hline
\end{tabular}

structure. The PMT used for the hygroscopic crystals, was also used by us in [5] and the tube used for the other is the same as the PMT-II tube of [4]. The experimental methods to record pulse height spectra of the scintillators with this PMT were described in [7]. The measured temperature of the scintillator is about $27 \pm 2{ }^{\circ} \mathrm{C}$ due to heat dissipation by the voltage divider. Specifying the measuring temperature is of relevance for scintillators like BGO that show strong temperature dependence of the scintillation yield. Light yield of BGO decreases by about $1.3 \%$ per degree Celsius [8].

For absolute scintillation yield determination we need to know: 1) the light collection efficiency of scintillation photons on the PMT photocathode, 2) the effective quantum efficiency of that PMT, 3) the absolute number of photoelectrons emitted by the photocathode in a scintillation event of known gamma ray energy, and 4) the photoelectron collection efficiency on the first dynode. Below these four items are further addressed in reversed order.

There are no means available to easily determine the photoelectron collection efficiency in PMTs. We therefore selected a PMT with a box-and-grid type dynode structure. This type of PMTs have collection efficiency close to $100 \%$ [9] and [10].

The total number of photoelectrons emitted by the photocathode in a scintillation event is determined by the method of Bertolaccine et al. [11]. The channel of the ${ }^{137} \mathrm{Cs} 662 \mathrm{keV}$ full energy peak in pulse height spectra is compared with the channel corresponding to the mean value of the so-called single photoelectron response (SER). Fig. 1 shows a typical SER curve on a log-linear scale measured with the Hamamatsu R1791 PMT at $-700 \mathrm{~V}$ cathode potential. A pulsed laser source was used to generate the single photoelectrons. In [4] we used the maximum of the response as the mean value but this is not a correct method. One should use the method of Dossi et al. [12] based on the mean value of the SER that we also adopted in [5]. The SER pulse height spectrum of Fig. 1 is fitted with a Gaussian function that describes the pulse heights from those photoelectrons that undergo the full multiplication of the PMT (curve 1). An exponential function (curve 2) describes the small amplitude signals

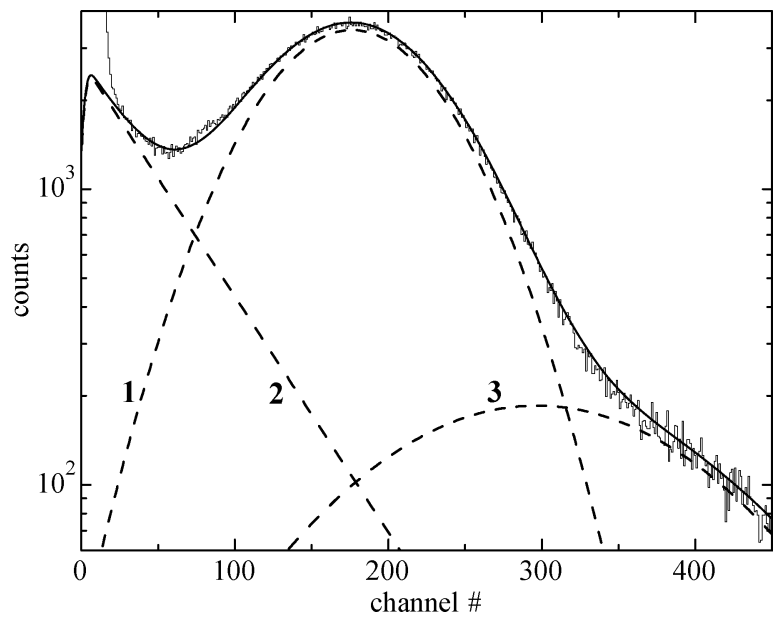

Fig. 1. SER pulse height spectrum with the R1791 PMT at $-700 \mathrm{~V}$ cathode voltage fitted with one exponential (curve 2) and two Gaussian curves (curve 1 and 3). The total fit is the solid line through the measured SER. Below channel 20 , the channel count increases steeply due to electronic noise of the amplifiers.

due to photoelectrons inelastically back-scattered from the first dynode [13]. These photoelectrons do not undergo the full multiplication process. In addition a Gaussian contribution (curve 3) can be seen due to two single photoelectrons producing two output signals within the shaping time of the electronic shaping amplifier.

The fit of the three functions to a measured SER is shown as the solid curve in Fig. 1. The mean channel position of the events contributing to this solid curve appears at about $13 \%$ lower channel number than the channel position of the maximum. In other words when the gain is determined from this maximum position it will be underestimated by $13 \%$.

Normally the measurements were performed at $-700 \mathrm{~V}$ PMT voltages. To avoid gain non-linearity due to high anode peak currents when measuring intense and fast scintillation events, a lower PMT voltage was used, i.e., $-600 \mathrm{~V}$ for LSO:Ce, LYSO:Ce and $\mathrm{LaCl}_{3}: \mathrm{Ce}$ and $-500 \mathrm{~V}$ for $\mathrm{LaBr}_{3}: \mathrm{Ce}$. At $-500 \mathrm{~V}$ PMT voltage it becomes difficult to fit the measured SER to the model of Dossi et al. [12] to determine the gain. An additional method was used. First the PMT gain was determined employing the slow scintillator CsI:Tl at $-700 \mathrm{~V}$. Comparing the position of the $662 \mathrm{keV}$ photopeak recorded at $-700 \mathrm{~V}$ with that recorded at $-500 \mathrm{~V}$, a good estimate for the PMT gain at $-500 \mathrm{~V}$ is obtained. The values for this indirect and direct method of gain determination agreed within $\pm 2 \%$.

The quantum efficiency $\mathrm{QE}_{\mathrm{PMT}}(\lambda)$ of a PMT as specified by manufacturers is determined with a light source placed in front of the PMT. The PMT current is then measured as function of the photon wavelength. In this configuration, photons are incident perpendicular to the PMT. Part $R_{\mathrm{PMT}}$ of them is reflected. Once reflected those photons are lost for detection. Another part $T_{\mathrm{PMT}}$ is transmitted through the photocathode and is also lost. The absorbed fraction has a probability $\eta_{e}$ to create a photoelectron. One may write

$$
\mathrm{QE}_{\mathrm{PMT}}(\lambda)=\eta_{e}\left(1-R_{\mathrm{PMT}}(\lambda)-T_{\mathrm{PMT}}(\lambda)\right)
$$



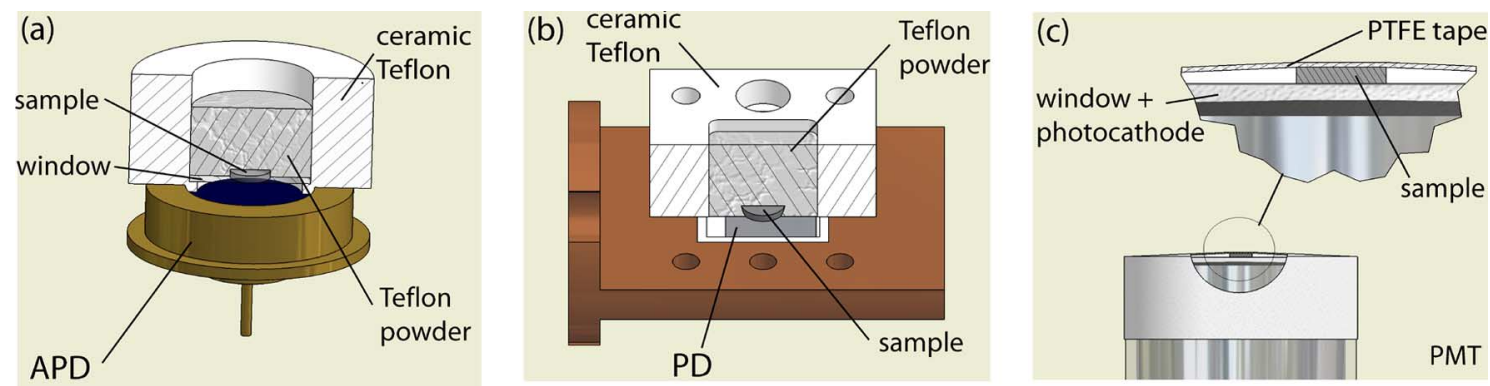

Fig. 2. Illustrations of reflector and scintillator mounting on (a) the APD, (b) the PD, and (c) the PMT. To compare the different sizes of the detectors the sample in the three pictures has the same dimension of $\varnothing 5 \times 1 \mathrm{~mm}$.

$\mathrm{QE}_{\mathrm{PMT}}(\lambda)$ is provided by the manufacturer, the detector reflectivity $R_{\mathrm{PMT}}(\lambda)$ was determined by us in [4]. $\eta_{e}$ and $T_{\mathrm{PMT}}$ are undetermined.

In the case of calibration of the scintillation yield, the scintillator (the light source) is placed on top of the PMT window and covered with a reflector in order to collect all scintillation photons on to the photocathode. In this configuration, photons that are reflected from the PMT will travel back through the scintillator and after one or more internal reflections may hit the PMT a second time, or a third time etc. In other words the photon has multiple chances to be detected, and effectively the quantum efficiency of detection will be larger than the one specified by the manufacturer. Suppose $R_{s}$ represents the effective average reflectivity of the scintillator that includes possible wavelength dependence and the fact that some photons require multiple reflections before entering the photocathode again. Since they travel an average path length $x$ there is a probability of self absorption. Suppose $T_{s}(x)$ is the effective transmission of the scintillator when a path length of $x$ is transferred. In equation form, the quantum efficiency $\mathrm{QE}_{s, d}$ for detection of scintillation light averaged over the emission profile of the scintillator can be given by

$$
\mathrm{QE}_{s, d}=\frac{\mathrm{QE}_{\mathrm{PMT}, \mathrm{av}}}{1-R_{s} T_{s}(x) R_{\mathrm{PMT}, \mathrm{av}}}
$$

where $\mathrm{QE}_{\mathrm{PMT}, \mathrm{av}}$ is the quantum efficiency specified by the manufacturer and $R_{\mathrm{PMT} \text {,av }}$ is the detector reflectivity; both averaged over the emission profile of the scintillator. The product $R_{s} T_{s}(x)$ is the probability that a photon reflected from the PMT reaches again the PMT window. This probability is optimized by placing an excellent reflector around the scintillator leading to $R_{s} \approx 1$ and by using small samples with negligible self absorption leading to small $x$ and $T_{s}(\mathrm{x}) \approx 1$.

To further enhance the light collection on the photocathode the scintillator is usually coupled to the PMT window by means of an optical coupling compound. Its use has important consequences. Without optical coupling only those photons incident on the PMT window within Brewster's angle may enter the window and are then able to generate photoelectrons. With ideal optical coupling Brewster's angle becomes $90^{\circ}$ and each photon from the sample incident on the PMT will enter the window and reach the photocathode. Although reflection at the photocathode-vacuum interface still occurs, the effective quantum efficiency is significantly enhanced. As mentioned in [4], a photon can be trapped within the PMT window by multiple reflections between the glass-air and cathode-vacuum interfaces. Each time it hits the photocathode it may be absorbed. For those photons actually $T_{\mathrm{PMT}}(\lambda)=0$ and $R_{\mathrm{PMT}}(\lambda)=0$ in (1) leading to enhancement of the PMT quantum efficiency. This phenomenon has been known for a long time [14] and is used to detect very faint light sources in astronomy. Finally, optical coupling brings $R_{s} T_{s}$ close to $100 \%$ because it requires fewer reflections and shorter average path length $x$ for the photon to re-enter the PMT window.

Quantum efficiency enhancement due to multiple reflections inside the PMT window and unknown dependence of QE on the angle of incidence on the photocathode may lead to effective quantum efficiencies quite different from the quantum efficiency specified for normal incidence of photons. The effective quantum efficiency becomes unspecified. This is the reason why we chose not to use optical coupling for calibrating the absolute scintillation light yield with PMTs. Photons then always enter the PMT window within Brewster's angle and in that case the quantum efficiency specified by the manufacturer best reflects the quantum efficiency experienced by the scintillation photons [15]. The disadvantage is the higher probability of photon trapping with photon losses in the scintillator, i.e., $R_{s} T_{s}<1$. To avoid this effect we used small crystals. In addition the samples have at least one unpolished surface in order to promote diffuse reflection.

\section{B. Si-(Avalanche) PhotoDiode Read Out}

An Advanced Photonix APD (type 630-70-72-510) and a Hamamatsu PIN-photodiode (type S3590-18) were used to characterize the scintillators with Si-based photon detectors. The delicate surface of the Advanced Photonix APD was protected against damaging by a quartz window positioned about $1 \mathrm{~mm}$ above the surface as illustrated in Fig. 2(a). The Hamamatsu PIN-photodiode has an epoxy resin window, and the scintillator crystal was mounted directly on the epoxy window as illustrated in Fig. 2(b).

For the absolute scintillation yield determination we need to know: 1) the light collection efficiency from the scintillator on the (A)PD, 2) the effective quantum efficiency of that (A)PD, and 3) the absolute number of electron-hole pairs created in a scintillation event of known gamma ray energy.

By comparing the channel number of the $662 \mathrm{keV}$ full-energy scintillation peak in pulse height spectra with the channel number corresponding with the signal produced by $17.8 \mathrm{keV}$ 
$\mathrm{X}$-rays of ${ }^{241} \mathrm{Am}$ detected directly in the (A)PD, the absolute electron-hole pair yield $Y_{\mathrm{eh}}$ can be obtained. It requires $3.67 \mathrm{eV}$ to produce one electron-hole pair in $\mathrm{Si}$ [16]. A $17.8 \mathrm{keV} \mathrm{X-ray}$ therefore produces $4850 \mathrm{eh}$-pairs, and that then calibrates the scintillation response to $662 \mathrm{keV}$. We used a $1700 \mathrm{~V}$ bias for the APD. By limiting the APD gain to below $100 \times$ and by using $17.8 \mathrm{keV}$ X-rays for calibration, the APD gain for electron-hole pairs created by photons is about the same as for electron-hole pairs created by low-energy X-rays [17]. To avoid gain drift, the APD was Peltier stabilized to $15 \pm 0.1^{\circ} \mathrm{C}$. The scintillator mounted on the quartz window was not in direct contact with the cooled APD, and we estimated about $20 \pm 2{ }^{\circ} \mathrm{C}$ for the scintillator temperature, i.e., a few degrees below room temperature. The temperature of the scintillator and PIN-photodiode was not controlled and is around room temperature $22 \pm 2{ }^{\circ} \mathrm{C}$.

The quantum efficiency of an (A)PD as specified by the manufacturer is determined by methods similar to that employed for PMT calibration. Like for PMTs the $\mathrm{QE}_{(\mathrm{A}) \mathrm{PD}}$ is reduced by reflection losses $R_{\mathrm{Si}}$ at the detector interface, and in addition by the smaller than $100 \%$ transmission $T_{\mathrm{Si}}(x)$ of the so-called $\mathrm{Si}$ "dead layer" of $x \mathrm{~nm}$ thickness between the APD surface and the photon sensitive depletion layer of the (A)PD. The "dead layer" is caused by impurities and defects below the surface of the photodiode that form recombination centers for electrons and holes created by absorbed photons [18].

For a Si-based detector one may write

$$
\mathrm{QE}_{(\mathrm{A}) \mathrm{PD}}(\lambda)=T_{\mathrm{Si}}(\lambda, x)\left(1-R_{\mathrm{Si}}(\lambda)\right) .
$$

Equation (3) is not valid for wavelengths longer than $800 \mathrm{~nm}$ because then Si becomes transparent.

Because of the reflector placed around the scintillator (2) applies again (replacing $\mathrm{QE}_{\mathrm{PMT}}$ for $\mathrm{QE}_{(\mathrm{A}) \mathrm{PD}}$ and $R_{\mathrm{PMT}}$ for $R_{\mathrm{Si}}$ ), and when $R_{s} T_{s}(x) \approx 100 \%$ we may write for the effective quantum efficiency of the Si-detectors

$$
\mathrm{QE}_{s, d}(\lambda) \approx T_{\mathrm{Si}, \text { av }}(x)
$$

where $T_{\mathrm{Si} \text { av }}$ is the transmission of the $\mathrm{Si}$ dead layer averaged over the scintillator emission profile.

In [4] we showed that, unlike for the photoelectron yield with PMT readout, the electron-hole pair yield with (A)PD readout is not much affected by absence or presence of optical coupling. This suggests that indeed $R_{s} T_{s}(x) \approx 100 \%$.

\section{Scintillator Packaging}

In order to accurately determine an absolute light yield it is important that all the photons generated inside the scintillator enter the photon detector. One should therefore maximize the light collection and minimize the internal absorption of photons by the scintillator (self absorption).

We used two different types of reflector configurations around the scintillator; 1) several layers of $0.1 \mathrm{~mm}$ thick PTFE tape, and 2) PTFE powder (Dupont Teflon 701-N) loosely pressed around the scintillator. PTFE has excellent reflection properties [19] and [20] over the entire relevant wavelength range.
For the PMT measurements we used the so-called "umbrella method". Teflon tape is spanned over the crystal and the 2 inch diameter PMT window, see Fig. 2(c). The sides of the crystal are not covered with reflecting tape. Photons that escape the sample via these side faces or via reflection from the photocathode will be reflected by the Teflon "umbrella" to the photocathode. These photons do not necessarily need to re-enter the scintillator, and photon loss by self absorption does not occur in such a case. Effectively $R_{s} T_{s}(x)$ in (2) increases.

In the case of (A)PD readout the umbrella method can not be used because of space restrictions. In stead, the so-called "pressed powder" method is used. Teflon powder is poured in a ceramic Teflon "holder" around the scintillator. By pressing the powder inside the "holder" the reflecting powder is in close contact with all the faces of the sample except the one contacting the detector, see Fig. 2(a) and 2(b). For comparison we also used the pressed powder method with the PMT.

The reflectivity of Teflon depends on the morphology and density of the material. Teflon tape has ideal density and we will adopt an effective reflectivity $R_{s}=0.98$. Reflectivity of the pressed powder is also very high but possibly may depend slightly on pressing conditions.

\section{RESULTS AND DISCUSSIONS}

To determine the absolute scintillation yield, the quantum efficiency and reflectivity curves for the PMT and Si-detectors are needed. Fig. 3(a) curve 2) shows the typical quantum efficiency of Si PDs and APDs as specified by manufacturers together with, curve 1$)$, the transmission $T_{\mathrm{Si}}(\lambda, x)$ of a $x=$ $5 \mathrm{~nm}$ thick Si layer [21]. Note the large drop in transmission at wavelengths shorter than $400 \mathrm{~nm}$. A similar drop is observed in the quantum efficiency at the same wavelength clearly demonstrating that indeed the transmission of a Si "dead layer" affects the quantum efficiency. Especially $\mathrm{LaCl}_{3}: \mathrm{Ce}$ and $\mathrm{LaBr}_{3}$ :Ce that emit at wavelengths below $400 \mathrm{~nm}$ have a poor detection efficiency with (A)PDs.

Curve 1) in Fig. 3(b) shows the quantum efficiency curve $\mathrm{QE}_{\mathrm{PMT}}(\lambda)$ for the R1791 Hamamatsu PMT as determined by the manufacturer on our special request. Curve 2) shows the reflectivity curve $R_{\mathrm{PMT}}(\lambda)$ of that same PMT as measured by us in [4]. Note that there is an anti-correlation between $R_{\mathrm{PMT}}(\lambda)$ and $\mathrm{QE}_{\mathrm{PMT}}(\lambda)$ especially around $460 \mathrm{~nm}$ and $520 \mathrm{~nm}$ which clearly demonstrates that quantum efficiency is determined by reflectivity as expressed by (1).

Fig. 3(c) and 3(d) show the X-ray excited emission spectra of the scintillators used for this work. The emission spectra were corrected for the monochromator transmission and photon detection efficiency of the X-ray facility. We assume that the emission under $\mathrm{X}$-ray excitation is the same as under gamma ray excitation. The average quantum efficiency $\mathrm{QE}_{\mathrm{PMT}, \mathrm{av}}$ and average reflectivity $R_{\mathrm{PMT} \text {,av }}$ calculated from the scintillator emission spectrum and the $\mathrm{QE}_{\mathrm{PMT}}(\lambda)$ and $R_{\mathrm{PMT}}(\lambda)$ curves in Fig. 3(b) are compiled in Table II.

The broad emission bands of BGO and CsI:Tl are not efficiently detected by the PMT because of relatively small quantum efficiency for wavelengths longer than $500 \mathrm{~nm}$. Those scintillators have the smallest values for $\mathrm{QE}_{\mathrm{PMT}, \mathrm{av}}$ The other 


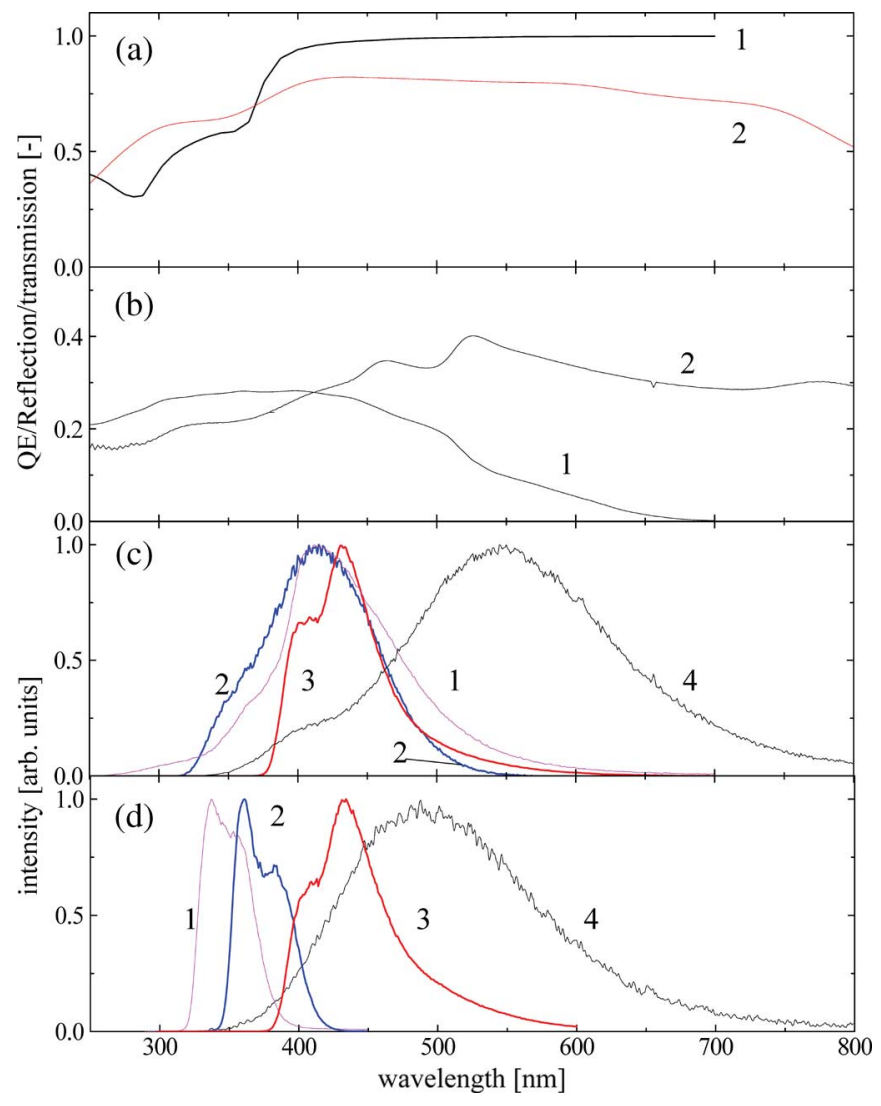

Fig. 3. a) Shows 1) the transmission of a $5 \mathrm{~nm}$ thick Si layer and 2) the quantum efficiency of the APD type 630-70-72-510. b) Shows 1) the R1791 PMT quantum efficiency measured by the manufacturer and 2) its reflectivity. $\mathrm{X}$-ray excited emission spectra of the scintillators in c) are 1) CsI:Na 2) NaI:Tl, 3) LYSO:Ce and 4) CsI:Tl and in d) are 1) $\mathrm{LaCl}_{3}: \mathrm{Ce}$, 2) $\mathrm{LaBr}_{3}$ :Ce, 3)LSO:Ce, and 4) BGO.

scintillators each have their emission near the maximum sensitivity of the PMT. Also note that a quartz window PMT is necessary to measure all emission from $\mathrm{CsI}: \mathrm{Na}, \mathrm{LaCl}_{3}: \mathrm{Ce}$, and $\mathrm{LaBr}_{3}: \mathrm{Ce}$.

The photoelectron yields $Y_{\text {phe }}$ expressed as the number of created photoelectrons in the PMT per MeV of absorbed gamma ray energy using the umbrella and pressed-powder methods are compiled in columns 4 and 6 of Table II. The electron-hole pair yields $Y_{\text {eh }}$ per MeV obtained with the Si-APD and the Si-PD using the pressed-powder method only are compiled in columns 8 and 10 of Table II.

The absolute scintillation yield $Y_{\mathrm{ph}}$ in photons per $\mathrm{MeV}$ of absorbed gamma ray energy is given by

$$
Y_{\mathrm{ph}}=\frac{Y_{\mathrm{phe} / \mathrm{eh}}}{R_{s}^{*} T_{s}^{*}\left(x^{*}\right) \mathrm{QE}_{s, d}}
$$

where $Y_{\text {phe/eh }}$ refers to photoelectron yield in the case of PMT detection and electron hole pair yield in the case of (A)PD detection. $\mathrm{QE}_{s, d}$ is given by (2) and (4). $R_{s}^{*}$ and $T_{s}^{*}$ are similar to $R_{s}$ and $T_{s}$ defined before but refer to reflection and self absorption losses of the scintillation photons before they first hit the detector. Neglecting self absorption expressed by $T_{s}$ and $T_{s}^{*}$ and assuming $R_{s}$ and $R_{s}^{*}=0.98$, the values for the scintillator yields $Y_{\mathrm{ph}}$ with PMT readout as compiled in columns 5 and 7 of Table II are obtained.

The error in the scintillation yield measured with the PMT is determined by errors in the mean value for the SER, errors in the measurements of the emission spectra, errors in the measured reflectivity of the photocathode, and errors in the quantum efficiency curve provided by the manufacturer. In addition we have to deal with unknown $R_{s} T_{s}$ and $R_{s}^{*} T_{s}^{*}$. The error in scintillation yield measured with the PMT is estimated $\pm 10 \%$. A first inspection of the results with the PMT shows that the umbrella method of reflector configuration provides about $10 \%$ higher

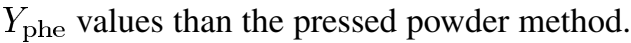

An important advantage of the APD and PD results over the PMT results is the fact that the intrinsic quantum efficiency of the (A)PD is much better defined and close to $100 \%$ when $\lambda>$ $400 \mathrm{~nm}$. In addition the reflectivity of the (A)PD is smaller and the correction for the back reflection by the scintillator covering will be less. The measured electron-hole pair yield only needs to be corrected for $R_{s}^{*} T_{s}^{*}$ and $T_{\mathrm{Si} \text {,eff }}(x)$. The error in the electronhole pair yield measured with the APD or PD is estimated $\pm 5 \%$ which is mainly determined by the error in the gain $( \pm 3 \%)$.

We may distinguish two different classes of scintillators: scintillators with a main emission above $400 \mathrm{~nm}$ and those with a main emission below $400 \mathrm{~nm}$.

\section{A. Emission $>400$}

The main part of the emission spectrum is at wavelengths longer than $400 \mathrm{~nm}$, which applies to CsI:Tl, BGO, LSO:Ce, and LYSO:Ce. For these scintillators transmission losses through the $\mathrm{Si}$ "dead layer" of the APD and PD are very small. $Y_{\mathrm{eh}}$ obtained with the APD are for all four scintillators practically the same as that obtained with the PD. The values also agree very well with the scintillation yields when using the umbrella method with PMT readout.

\section{B. Emission $<400$}

A large part of the emission spectrum is at wavelengths shorter than $400 \mathrm{~nm}$ which applies to $\mathrm{LaCl}_{3}: \mathrm{Ce}, \mathrm{LaBr}_{3}: \mathrm{Ce}$, $\mathrm{YAlO}_{3}: \mathrm{Ce}$, and $\mathrm{CsI}: \mathrm{Na}$. Table II shows that $Y_{\text {eh }}$ obtained with the PD is less than that obtained with the APD and the difference increases with shorter average wavelength of emission. This strongly suggests that the Si "dead layer" in the PD is thicker than in the APD. By choosing thicknesses of $x=5 \mathrm{~nm}$ and $x=2 \mathrm{~nm}$ and correcting for the appropriate $T_{\mathrm{Si} \text {,eff }}(x)$ values the scintillation yield $Y_{\mathrm{ph}}$ values obtained with the PD and APD agree very well, see column 9 and 11 of Table II.

One may question whether the difference in yield obtained with the PMT umbrella method and the PMT pressed-powder method is due to differences in $R_{s}$ and $R_{s}^{*}$. With the umbrella method photons can escape from the scintillator via its side faces. These photons are reflected by the umbrella towards the photocathode. Effectively the path length $x$ through the scintillator is shorter leading to values for $T_{s}(x)$ closer to $100 \%$. This may explain the lower $Y_{\mathrm{ph}}$ values obtained with the PMT pressed powder method. The reflectivity of the (A)PD is lower than that of the PMT. We then expect that although the pressed powder method was used, photon losses by trapping inside the scintillator is smaller than in the case of the PMT 
TABLE II

Absolute Scintillation Yield $Y_{P H}(\mathrm{ph} / \mathrm{MeV})$, Photoelecton Yields $Y_{\mathrm{PhE}}(\mathrm{phe} / \mathrm{MeV})$ and Electron Hole Pair Yields $Y_{\mathrm{EH}}$ (eh-pairs/MeV) Determined With A PMT, APD, AND PD. PD RESUlts BETWEEN BRACKETS ARE From MosZYNSKi et al. (REF. [3]) AND

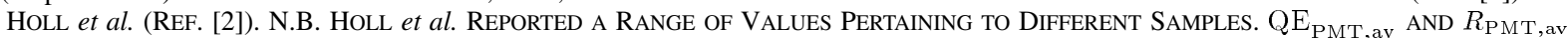
ARE THE PMT QUANTUM EFFICIENCY AND REFLECTIVITY AVERAGED OVER THE EMISSION PROFILE OF THE SCINTILLATOR

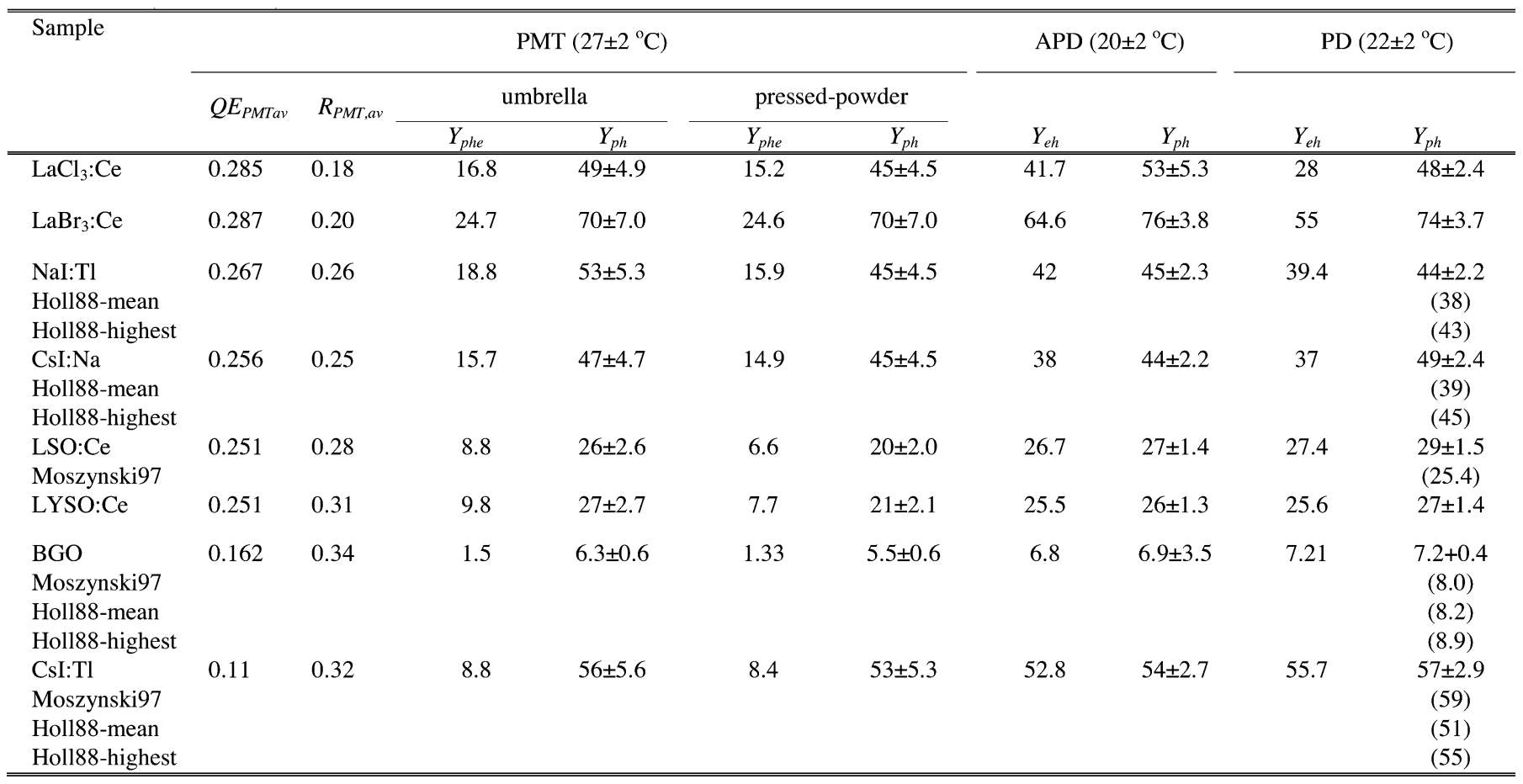

pressed powder method. Our observation in [4] that for APD readout the ratio in electron-hole pair yield measured with and without optical coupling is close to 1 confirms this.

We regard the $Y_{\mathrm{ph}}$ values obtained with the APD and PD as the most reliable values for the absolute scintillation yield of the studied samples. Possible errors arise from unknown amount of self absorption, errors in the gain determination and possible underestimated values for reflection losses $R_{s}$ and $R_{s}^{*}$. We estimate that the overall error can be $5 \%$. We therefore regard the values for $Y_{\mathrm{ph}}$ in columns 9 and 11 of Table II as the minimum absolute intrinsic scintillation yield of the studied samples. The yields obtained with the PMT are subject to larger errors because quantum efficiency and light collection efficiency are poorly defined. Effects of photon trapping in the PMT window, quantum efficiencies that depend on the angle of incidence on the photocathode, photoelectron collection efficiencies on the first dynode, and gain determination are all difficult to account for properly.

Because the method of yield determination with PMTs adopted in this work is different than that used by others and because of the inherent difficulties associated with calibrations using PMTs, it is not feasible to properly compare our results with that of others. This problem does not arise for (A)PD readout and a comparison with the early results by Holl et al. [2] and by Moszyński et al. [3] can be made.

In Table II we provided the PD yield numbers reported by Holl et al. [2] and by Moszyński et al. [3]. Generally, the highest scintillation yield measured by [2] and the data from [3] compare well with our data. The somewhat higher value that we measured for LSO:Ce may be related to the better quality of our
LSO:Ce scintillator. The scintillation yield of BGO strongly depends on shape, size, and temperature of the sample, and in that sense the $10 \%$ smaller yield that we find for BGO is not too worrying.

\section{CONCLUSIONS}

With three types of photon detectors we determined the absolute scintillation yield of a set of eight different scintillators. For the calibration with a PMT we used a different method then commonly; a correction for the PMT reflectivity was made and the mean value for the single photoelectron response pulse height spectrum was used for PMT gain calibration instead of the maximum in the single photoelectron response spectrum. Fortunately, the two new corrections largely cancel each other and the yields obtained with our method do not differ dramatically from values reported by us and others using the "old" method. Nevertheless, we are of the opinion that the "new" method should be preferred. Despite all the corrections, yield determination with PMTs remains inherently cumbersome because the effective PMT quantum efficiency can not be properly defined, photoelectron collection efficiency can not be measured, and because of errors in gain determination. Most of these problems are absent in the case of APD and PD readout, and these sensors are therefore the ones to be preferred for determining the scintillation yield.

\section{REFERENCES}

[1] E. Sakai, "Recent measurements on scintillator-photodetector systems," IEEE Trans. Nucl. Sci., vol. 34, no. 1, pp. 418-422, Feb. 1987. 
[2] I. Holl, E. Lorenz, and G. Mageras, "A measurement of the light yield of common inorganic scintillators," IEEE Trans. Nucl. Sci., vol. 35, no. 1, pp. 105-109, Feb. 1988.

[3] M. Moszyński, M. Kapustra, M. Mayhugh, D. Wolski, and S. O. Flyckt, "Absolute light output of scintillators," IEEE Trans. Nucl. Sci., vol. 44, no. 3, pp. 1052-1061, Jun. 1997.

[4] J. T. M. de Haas, P. Dorenbos, and C. W. E. van Eijk, "Measuring the absolute light yield of scintillators," Nucl. Instrum. Methods, vol. A537, pp. 97-100, 2005.

[5] J. T. M. de Haas, P. Dorenbos, and C. W. E. van Eijk, "The absolute light yield of $\mathrm{LaBr}_{3}: 5 \% \mathrm{Ce}$ and $\mathrm{LaCl}_{3}: 10 \%$ Ce," in Proc. 8th Int. Conf. Inorganic Scintillators Their Use in Scientific Industrial Applications (SCINT), A. Gektin and B. Grinyov, Eds., Alushta, Crimea, Ukraine, Sep. 2005, pp. 135-137.

[6] M. Gierlik, M. Moszyński, A. Nassalski, A. Syntfeld-Każuch, T. Szczesśniak, and $Ł$. Świderski, "Investigation of absolute light output measurement techniques," IEEE Trans. Nucl. Sci., vol. 54, no. 4, pp. 1367-1371, Aug. 2007.

[7] P. Dorenbos, J. T. M. de Haas, and C. W. E. van Eijk, "Gamma ray spectroscopy with a $\varnothing 19 \times 19 \mathrm{~mm} 3 \mathrm{LaBr} 3: 0: 5 \% \mathrm{Ce} 3+$ scintillator," IEEE Trans. Nucl. Sci., vol. 51, no. 3, Jun. 2004.

[8] C. Rozsa, C. Grodsinsky, D. Penn, P. Raby, and R. Schreiner, "The change of gamma equivalent energy with temperature for scintillation detector assemblies," in Proc. IEEE Nucl. Sci. Symp., Oct. 1999, vol. 2, pp. 686-690.

[9] "Photomultiplier Tube. Principle to Application," Hamamatsu Photonics K.K. Electron Tube Center, Japan, 1994, p. 30.

[10] “Photomultiplier Handbook," Burle Industries, Inc., Tube Products Div., Lancaster, PA, 1989, p. 28.

[11] M. Bertolaccini, S. Cova, and C. Bussolati, "A technique for absolute measurement of the effective photoelectron per $\mathrm{keV}$ yield in scintillation counters," presented at the Nucl. Electr. Symp., Versailles, France, Sep. 1968.
[12] R. Dossi, A. Ianni, G. Ranucci, and O. J. Smirnov, "Methods for precise photoelectron counting with photomultipliers," Nucl. Instrum. Methods, vol. A451, pp. 623-637, 2000.

[13] "Photomultiplier Tubes. Principles and Applications," Photonis Imaging Sensors, Brive cedex, France, 2002.

[14] W. D. Gunter, E. F. Erickson, and G. R. Grant, "Enhancement of photomultiplier sensitivity by total internal reflection," Appl. Opt., vol. 4, pp. 512-513, 1965.

[15] D. Motta and S. Schönert, "Optical properties of Bialkali photocathodes," Nucl. Instrum. Methods, vol. A539, pp. 217-235, 2005.

[16] R. H. Pehl, F. S. Goulding, D. A. Landis, and M. Lenzlinger, "Accurate determination of the ionization energy in semiconductor detectors," Nucl. Instrum. Methods, vol. 59, pp. 45-55, 1968.

[17] M. Moszyński, M. Kapustra, M. Mayhugh, M. Balcerzyk, M. Szawlowski, and D. Wolski, "Large area avalanche photodiodes in X-rays and scintillation detection," Nucl. Instrum. Methods, vol. A442, pp. 230-237, 2000.

[18] A. M. Saad, "High quantum efficiency of UV-enhanced silicon photodiodes," Can. J. Phys., vol. 80, pp. 1601-1608, 2002.

[19] B. J. Pichler, E. Lorenz, R. Mirzoyan, L. Wiess, and S. I. Ziegler, "Production of a diffuse very high reflectivity material for light collection in nuclear detectors," Nucl. Instrum. Methods, vol. A442, pp. 333-336, 2000.

[20] V. R. Weidner and J. J. Hsia, "Reflection properties of pressed polytetrafluorethylene powder," J. Opt. Soc. Amer., vol. 71, no. 7, pp. 856-861, Jul. 1981.

[21] H. R. Philipp and E. A. Taft, "Optical constants of silicon in the region 1 to 10 eV," Phys. Rev., vol. 120, no. 1, pp. 37-38, Oct. 1960. 